DOI: $10.34185 / 1991-7848.2018 .01 .06$

УДК 621.385

С.В. Быткин, Т.В. Критская

\title{
МОДЕЛИРОВАНИЕ S-ОБРАЗНОГО ПРОЦЕССА НАКОПЛЕНИЯ \\ А- И Е-ЦЕНТРОВ В ИЗОВАЛЕНТНО ЛЕГИРОВАННОМ ГЕРМАНИЕМ КРЕМНИИ В СРЕДЕ STATISTICA И МАТНСАD
}

Кремний, легированный изовалентной примесью германия (Si<Ge> или SiGe в англоязычной научнотехнической литературе), обладает рядом физико-технологических преимуществ с точки зрения обеспечения радиационной стойкости прп- и прпр-структур. Применение именно такого кремния весьма перспективно для силовой электроники, поскольку он способен вытеснить используемый в настоящее время кремний традиционного качества, легированный фосфоом (Si $<P>)$ за счёт своей больщей термостабильности и радиационной стойкости, что положительно влияет на качество изготавливаемых на его основе приборов. Целью работы является моделирование в среде STATISTICA и MathCAD нелинейного накопления основных радиационных дефектов в кремнии и оценка возможности использования Gе как примеси, существенно замедляющей радиационную деградацию Si. Установлен процесс нелинейного накопления основных радиационных дефектов ( $A-u$ E- центров) в кремнии, подвергаемом облучению $\alpha$-частицами. Проведено моделирование этого процесса в среде STATISTICA и MathCAD. Показано, что легирование кремния германием до определённых концентраций позволяет существенно замедлить радиационную деградацию кремния и использовать такой материал для изготовления радиационностойких полупроводниковых приборов, в частности, многослойных структур высоковольтных силовых приборов общепромышленного и транспортного электропривода.

Ключевые слова: легирование кремния германием, нелинейное накопление основных радиационных дефектов, изготовление радиационно-стойких полупроводниковых приборов.

\section{Постановка проблемы в общем виде}

Необходимость обеспечения радиационной стойкости кремния, используемого для изготовления многослойных структур силовых приборов электропривода общепромышленного оборудования и транспортного электропривода в наземных условиях определяется отказами высоковольтных силовых приборов, вследствие действия вторичных космических лучей, образовавшихся в атмосфере [1].

\section{Анализ последних исследований и публикаций}

Кремний, легированный изовалентной примесью германия (Si<Ge> или $\mathrm{SiGe}$ в англоязычной научно-технической литературе), обладает рядом физико-технологических преимуществ с точки зрения обеспечения радиационной стойкости npn- и npnp-структур $[2,3,4]$. Применение именно такого кремния весьма перспективно для силовой электроники, поскольку он способен вытеснить используемый в настоящее время кремний традиционного качества, легированный фосфоом (Si<P>) за счёт своей большей термостабильности и радиационной стойкости, что положительно влияет на качество изготавливаемых на его основе приборов [5]. В Украине разработана промышленная технология выращивания монокристаллического $\mathrm{Si}<\mathrm{P}, \mathrm{Ge}>$ [6], что позволяет говорить о существовании потенциальной возможности его производства и экспорта в период до 2040 г [7], в том числе, для изготовления дискретных силовых приборов, мировой рынок которых оценивается в 2024 г. $\approx \$ 23$ млрд. (на \$10 млрд. больше, чем в 2014 г. [8]). Доля кремниевых приборов прогнозируется равной $\approx 87 \%$, что определятся ценовым преимуществом монокристаллического кремния среди прочих полупроводниковых материалов. Остальную часть рынка займут приборы на основе $\mathrm{SiC}$ и GaN. Полупроводниковый материал, используемый в технологии силовых полупроводниковых приборов (СПП), наряду с технологичностью и доступной ценой должен

(c) Быткин С.В., Критская Т.В., 2018 
обладать комплексом электрофизических и структурных свойств, которые могут привести к существенно новым характеристикам приборов. Например, обеспечить высокую радиационную стойкость СПП, задача создания которых принципиально не была решена при использовании монокристаллов стандартного качества. Фактически, такой материал является high-tech сырьевым материалом для изготовления изделий электронной техники (ИЭТ) $[9,10]$, т.е. таким, при производстве которого используются технологии, основанные на практическом применении фундаментальных научных знаний, например, о внутренних точечных дефектах, образующихся при производстве монокристаллов, их взаимодействии с примесями при радиационном и термическом воздействии и другими особенностями инженерии дефектов (defectengineering) выращиваемого кристалла [11].

\section{Выделение нерешённых научно-технических задач}

Особенности накопления радиационных дефектов в $\mathrm{Si}<\mathrm{P}>$ и $\mathrm{Si}<\mathrm{P}, \mathrm{Ge}>$, моделировались в [12] с использованием полученных в [13] экспериментальных результатов, однако в этих работах не учитывалось S-образное накопление дефектов.

Целью настоящей работы является моделирование в среде STATISTICA и MathCAD нелинейного накопления основных радиационных дефектов в кремнии и оценка возможности использования Ge как примеси, существенно замедляющей радиационную деградацию Si.

\section{Изложение основного материала.}

Облучение $\alpha$-частицами производилось на образцах монокристаллов кремния, выращенных по методу Чохральского, легированных фосфором, а также фосфором и германием, соответсвенно $\mathrm{Si}<\mathrm{P}>$ и $\mathrm{Si}<\mathrm{P}, \mathrm{Ge}>$. На рис. 1 (а, б) приведен характер зависимости накопления А- центра в контрольных образцах $\mathrm{Si}<\mathrm{P}>$ и $\mathrm{Si}<\mathrm{P}, \mathrm{Ge}>$, аппроксимированных уравнениями в среде STATISTICA.

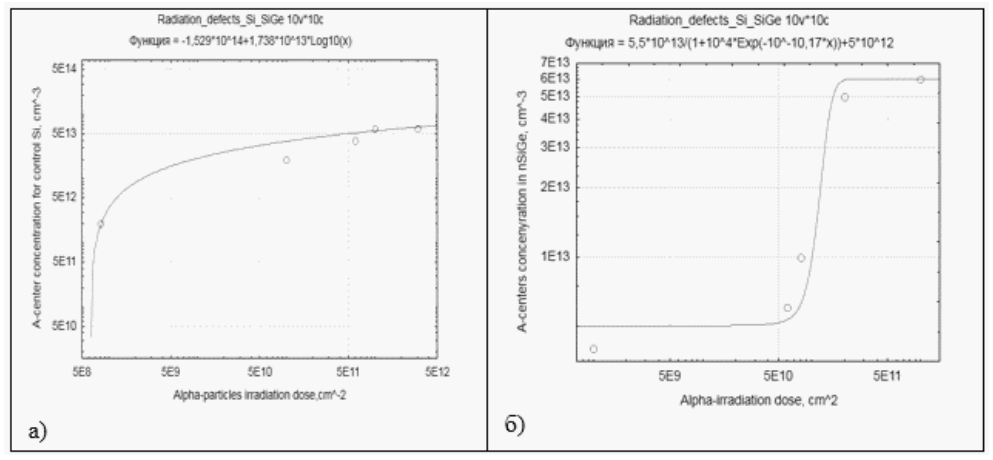

Рисунок 1 - Экспериментальные данные по накоплению А - центров в контрольном образце $\mathrm{Si}<\mathrm{P}>$, (а) и в $\mathrm{Si}<\mathrm{P}, \mathrm{Ge}>$, (б) в зависимости от дозы облучения $\alpha$-частицами $\mathrm{D}(\mathrm{t})$

$\mathrm{S}$-образный характер накопления Е-центров в $\mathrm{Si}<\mathrm{P}, \mathrm{Ge}>$ также является особенностью этого материала (рис.2, а, б).

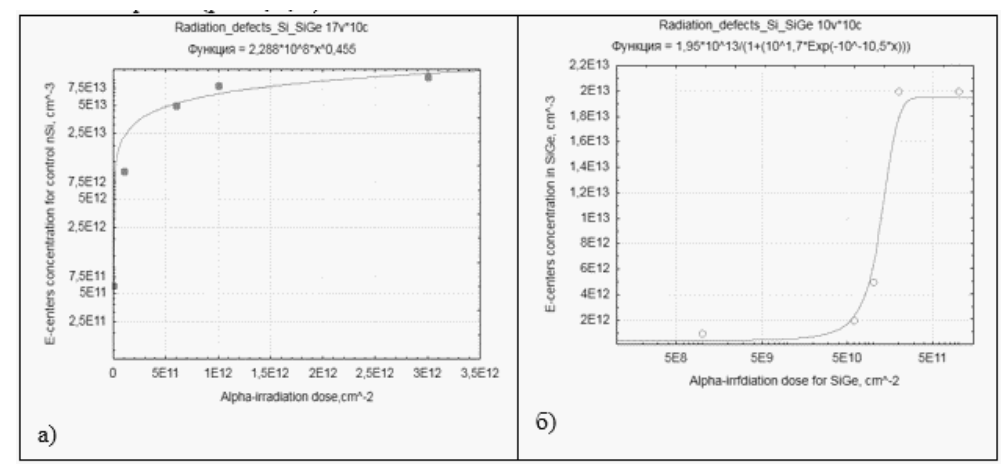

Рисунок 2 - Экспериментальные данные по накоплению $\mathrm{E}$ - центров в контрольном образце $\mathrm{Si}<\mathrm{P}>$, (а) и в $\mathrm{Si}<\mathrm{P}, \mathrm{Ge}>$, (б) в зависимости от дозы облучения $\alpha$-частицами $\mathrm{D}(\mathrm{t})$ 
Для $\mathrm{D}(\mathrm{t}) \leq 1 \cdot 10^{11} \mathrm{~cm}^{-2}$ наблюдается существенное замедление накопления А- и Е-центров в $\mathrm{Si}<\mathrm{P}, \mathrm{Ge}>$, что определяется, скорее всего, стоками вакансий, связанными с изовалентной примесью. При больших дозах облучения происходит выравнивание (А-центры) концентраций этих дефектов, рис. 3.

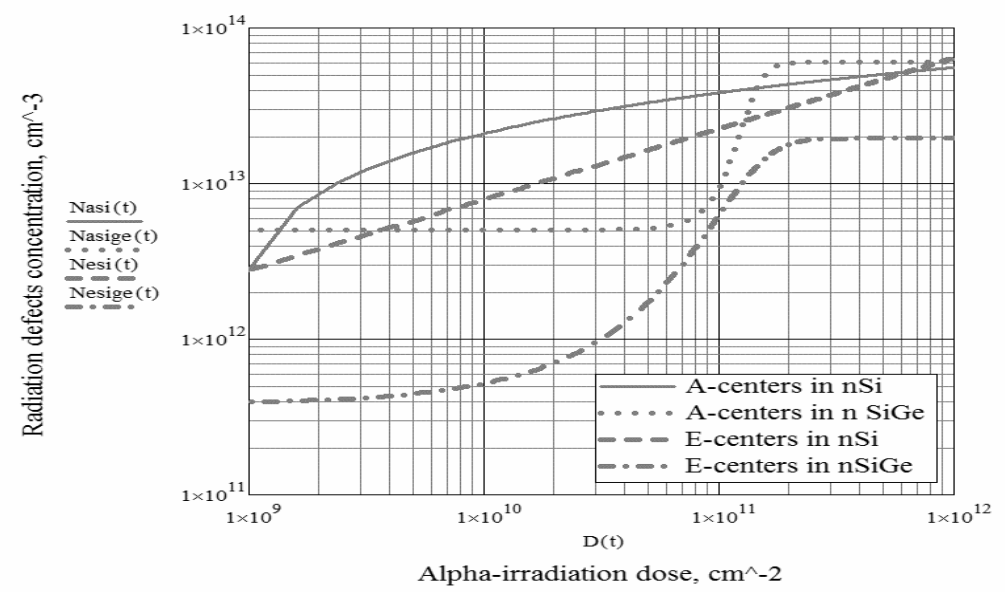

Рисунок 3 - Дозовая зависимость образования А- и Е-центров в $\mathrm{Si}<\mathrm{P}>, \mathrm{Si}<\mathrm{P}, \mathrm{Ge}>$ (аппроксимирующие зависимости для модели расчёта вероятности образования радиационных дефектов, здесь и далее $\Phi \alpha \equiv \mathrm{D}(\mathrm{t}))$

Нелинейный характер накопления дефектов в $\mathrm{Si}<\mathrm{P}, \mathrm{Ge}>$ связан с различными скоростями их введения при различных дозах облучения $\alpha$-частицами $\Phi_{\alpha}$, рис.4.

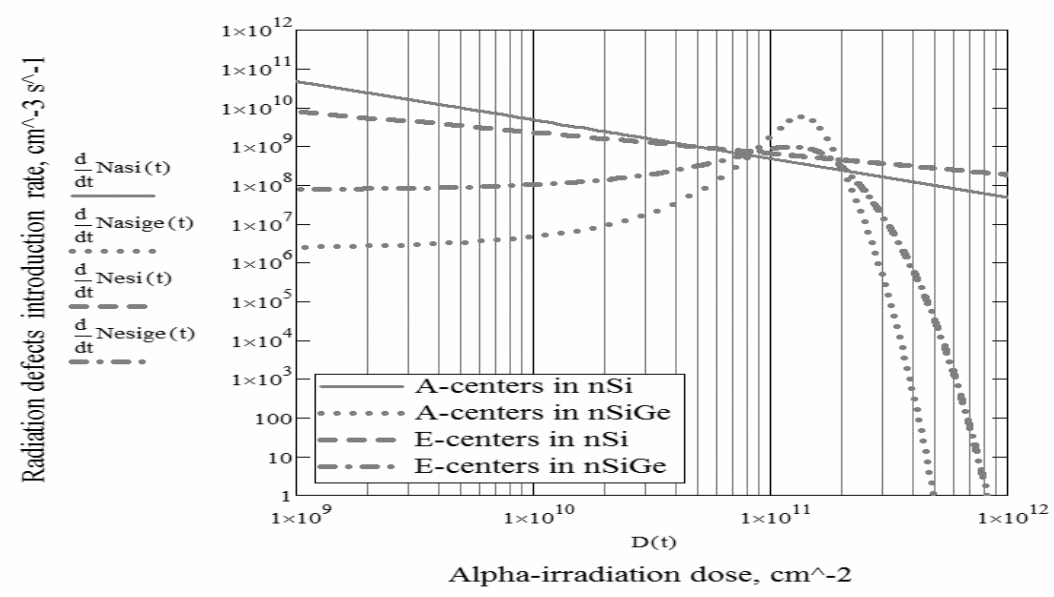

Рисунок 4 - Зависимость скорости накопления радиационных дефектов

в $\mathrm{Si}<\mathrm{P}>$ и $\mathrm{Si}<\mathrm{P}, \mathrm{Ge}>$ от экспозиционной дозы $\alpha$-облучения

В частности, при $\Phi_{\alpha} \leq 10^{10} \mathrm{~cm}^{-2}$ скорость генерации А- и Е-центров в кремнии, легированном $\mathrm{Ge}$, на несколько порядков ниже, чем в контрольных образцах, при $10^{10} \mathrm{~cm}^{-2} \leq \Phi_{\alpha} \leq 2 \cdot 10^{11} \mathrm{~cm}^{-2}$ в $\mathrm{Si}<\mathrm{P}, \mathrm{Ge}>$ наблюдается активное накопление этих центров со скоростью, равной (Е-центры) или большей (А-центры), чем в не легированном германием кремнии. При $\Phi_{a} \geq 2 \cdot 10^{11} \mathrm{~cm}^{-2}$ эти дефекты практически не образуются.

Полученные зависимости качественно могут быть объяснены простой схемой [14]. При облучении $\mathrm{Si}<\mathrm{P}, \mathrm{Ge}>$ образуется устойчивый кластер $\mathrm{GeVO}$, уменьшающий концентрацию вакансий в облучаемом материале и, как следствие, концентрацию радиационных дефектов на начальном этапе облучения. Эффективность процесса формирования стока для вакансий достигается за счёт коррелированного распределения германия и кислорода, приводящего к более полной компенсации внутренних упругих напряжений в кремнии $[15,16,17]$. Сток вакансий, образующих кластер, при $\Phi_{\alpha} \leq 1 \cdot 10^{11} \mathrm{~cm}^{-2}$ уменьшает вероятность образования 
A- и Е-центров в $\mathrm{Si}<\mathrm{P}, \mathrm{Ge}>$ по сравнению с контрольным $\mathrm{Si}<\mathrm{P}>$. После насыщения комплекса $\mathrm{Ge}-\mathrm{O}$ вакансиями активизируется процесс образования А-центров, достигая максимума при $\Phi_{\alpha} \approx 1 \cdot 10^{11} \mathrm{~cm}^{-2}$. Фактически, увеличение вероятности образования А-центров связано с взаимодействием междоузельного кислорода $\mathrm{O}_{\mathrm{i}}$, окружающего атомы $\mathrm{Ge}$, с вакансиями, вводимыми в материал облучением. После исчерпания $\mathrm{O}_{\mathrm{i}}$ в ходе квазихимической реакции вероятность образования комплексов $\mathrm{V}-\mathrm{O}$ в $\mathrm{Si}<\mathrm{P}, \mathrm{Ge}>$ существенно ниже, чем в контрольном материале. Это и определяет наблюдаемое в [18] замедление деградации $\tau_{\mathrm{p}}$ в $\mathrm{Si}<\mathrm{P}, \mathrm{Ge}>$ при больших дозах облучения. Эффективность введения, или, иными словами, количество образовавшихся A- и Е-центров в $\mathrm{Si}<\mathrm{P}, \mathrm{Ge}>$ на начальном этапе облучения, т.е. при ещё не насыщенных стоках для вакансий $\mathrm{Ge}-\mathrm{O}_{\mathrm{i}}$ существенно ниже, чем в контрольных образцах, что показано на рис. 5 .

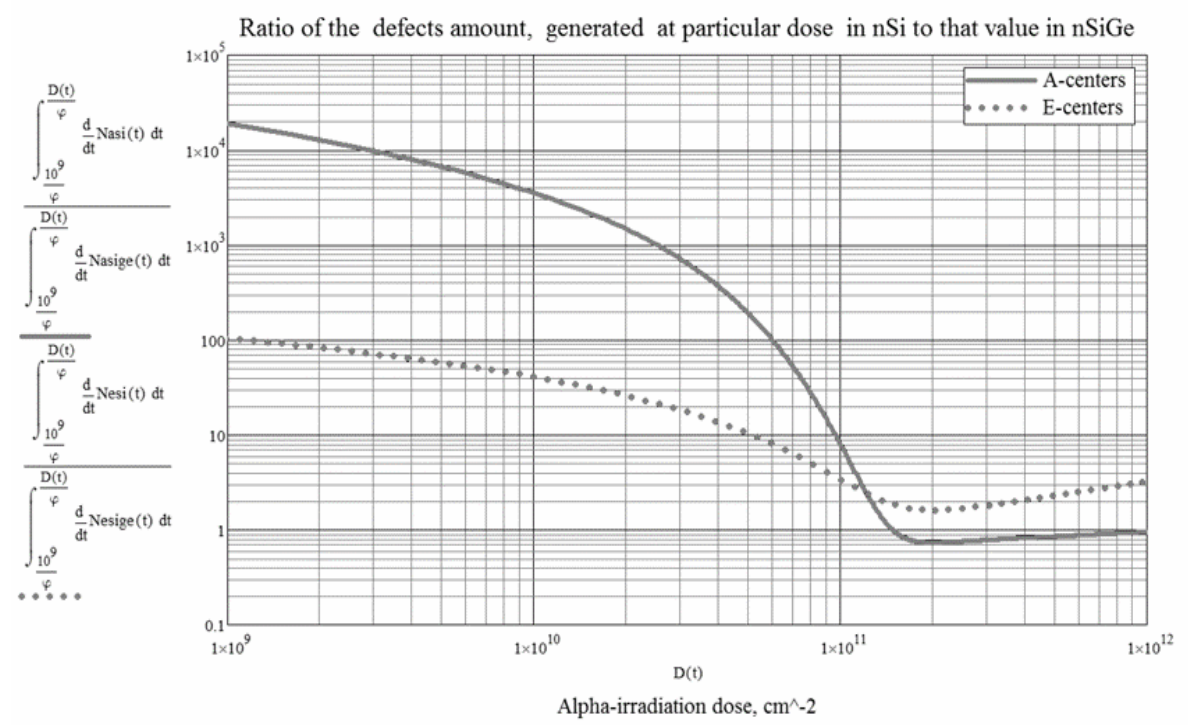

Рисунок 5 - Относительная эффективность накопления А- и Е-центров при различных дозах $\alpha$-облучения

Оценка эффективности применения кремния, легированного изовалентной примесью, при облучении в различных диапазонах доз может быть проведена с использованием стандартной формулы для среднего значения функции, приведенной на рис. 5, на определённом интервале значений аргумента, в данном случае - флюенсов $\alpha$-облучения: где $\mathrm{D}(\mathrm{t})$-текущее значение дозы $\alpha$-облучения, Dinitial-начальное значение, в данном случае $10^{9} \mathrm{~cm}^{-2}$.

Аналогично проводится расчёт для Е-центров.

Результаты приведены в табл. 1.

Таблица 1

Влияние дозы $\alpha$-облучения $\Phi_{\alpha}$ на накопление радиационных дефектов в nSi иnSiGe (концентрация $\mathrm{Ge} \approx 5 \cdot 10^{19} \mathrm{~cm}^{-3}$ )

\begin{tabular}{|l|l|l|l|l|l|}
\hline$\Phi_{\alpha}$, см $^{-2}$ & $10^{10}$ & $10^{11}$ & $10^{12}$ & $10^{13}$ & $10^{14}$ \\
\hline $\begin{array}{l}\text { Относительное превышение концентрации } \\
\text { А-центров в Si над этой величиной в SiGe }\end{array}$ & $3,527 \cdot 10^{3}$ & 7,963 & 0,948 & 1,264 & 1,58 \\
\hline То же для Е-центров & 40,703 & 3,377 & 3,218 & 9,444 & 27,191 \\
\hline
\end{tabular}

Следовательно, легирование кремния Ge с концентрацией $\approx 5 \cdot 10^{19} \mathrm{~cm}^{-3}$ при облучении в диапазоне $10^{9} \leq \Phi_{\alpha} \leq 10^{10} \mathrm{~cm}^{-2}$ позволяет в среднем в $10^{3}$ раз снизить количество образующихся А-центров. Эффективность замедления образования Е-центров существенно ниже во всём диапазоне доз. 


\section{Выводы из проведенного исследования и перспективы дальнейшего поиска}

Процессы радиационного дефектообразования в кремнии, легированном германием, определяющие стабильность его электрофизических свойств в условиях действия ионизирующего облучения, носят сложный характер, эффективность протекания этих процессов нелинейно связана с интегральной дозой облучения. Возможность использования $\mathrm{Si}<\mathrm{P}, \mathrm{Ge}>$ для повышения радиационной стойкости многослойных npn, npnp структур может быть подтверждена только изготовлением тестовых приборов на $\mathrm{Si}$ с различной концентрацией легирующего германия и контрольного $\mathrm{Si}<\mathrm{P}>$ с проведением их испытаний [19].

\section{ЛИТЕРАТУРА}

1. Munaf Rahimo. Power Semiconductors for Power Electronics Applications ABB Switzerland Ltd, Semiconductors. CAS-PSI Special course Power Converters, Baden Switzerland, 8th May 2014, slides 43, 46.

2. Bytkin S.V. Silicon Doped with Germanium (n-Si<Ge $>$ ) Usage For Manufacturing Of Radiation Hardened Devices And Integrated Circuits. Fourth European Conference on Radiation and Its Effects on Components and Systems Proceedings, 1997. Cannes, France, pp. 141-146.

3. Быткин С.В., Критская Т.В., Радин Е.Г., Гончаров В.И., Куницкий Ю.И., Кобелева С.П. Экспериментальное исследование характеристик тиристоров, изготовленных на $\mathrm{Si}<\mathrm{Ge}>$, при действии гамма-облучения. Известия высших учебных заведений. Материалы электронной техники. №3, 2012, стр. 45-48.

4. S. V. Bytkin, T. V. Kritskaya, S. P. Kobeleva. Statistical analysis of germanium influence on radiation and thermal stability of the n-p-n-p device structures based on CZ-Si 〈P, Ge〉 electrophysical properties. Russian Microelectronics, December 2014, Volume 43, Issue 8, pp. 546-551.

5. S. Taranovich. Si vs. GaN vs. SiC: Which process and supplier are best for my power design? Сайт EDN, March 15, 2013. [Электронный ресурс] Режим доступа / http://www.edn.com/design/powermanagement/4409627/3/Si-vs--GaN-vs--SiC--Which-process-and-supplier-are-best-for-my-power-design.

6. Критская Т. В. Современные тенденции получения кремния для устройств электроники: монография / Т. В. Критская; Запорож. гос. инженер. акад. - Запорожье: ЗГИА, 2013. - 353 с.

7. Crystal Growth Technology. [Электронный ресурс] Режим доступа / https://www.google.com.ua/url?sa=t\&rct=j\&q=\&esrc=s \&frm=1\&source=web\&cd=2 \&cad=rja \&uact=8\&ved=0CC YQFjABahUKEwicjvyjgJrJAhXGCCwKHX4eBQQ\&url=https\%3A\%2F\%2Fwww.jsap.or.jp\%2Fenglish\%2Fimages\%2 Facademic_roadmap\%2Farm_e_09.pdf\&usg=AFQjCNGIxTwXP53r7IiRQ5U3f-

E_rvbK4A\&bvm=bv.107763241,d.bGg.

8. R. Pell. Power Week: Si-Based Power Discretes to Continue to Dominate Over Next Decade. Сайт EETimes 11/26/2014. [Электронный ресурс] Режим доступа / http://www.eetimes.com/document.asp?doc_id=1324777\&. 9. Когнитивный вызов и информационные технологии / Г.Г. Малинецкий [и др.] // Препринты ИПМ им. M.В. Келдыша. 2010. № 46. 28 с. URL: http://library.keldysh.ru/preprint.asp?id=2010-46.

10. Жукова Е.А. Hi-Tech: феномен, функции, формы. / Под ред. И.В. Мелик -Гайказян. - Томск: Издательство Томского государственного педагогического университета, 2007. - 376 с.

11. G. Fisher, M. R. Seacrist, R. W. Standley. Silicon Crystal Growth and Wafer Technologies. ProceedingsoftheIEEE, Vol. 100, May 13th, 2012, 1454-1474.

12. Быткин С. В., Критская Т.В. Моделирование процесса накопления радиационных дефектов в процессе облучения монокристаллов $\mathrm{Si}<\mathrm{P}>$ и $\mathrm{Si}<\mathrm{P}, \mathrm{Ge}>$. Металургія. Збірник наукових праць Запорізької державної інженерної академії, вип.1(21). - Запоріжжя, 2010. - С.116-125.

13. BytkinS.V. Reduction of the Thermostable Radiation Defects Probability Formation in Si and SiGe as a Physical Basis of the Bipolar npn Transistors Radiation Hardness Increase at the Application of the Radiation \& Thermal Processing (RTP- technology). MAPLD 2004 Proceedings, September 8-10, 2004. The Johns Hopkins University- Applied Physics Laboratory-Laurel, Maryland, USA. [Электронный ресурс] Режим доступа / http://klabs.org/mapld04/papers/p/p138_bytkin_p.doc.

14. C.A. Londos, E. N. Sgourou, A. Chroneos, V. V. Emtsev. Carbon, oxygen and intrinsic defect interactions in germanium-doped silicon. Semicond. Sci. Technol. 26(2011) 105024 (7pр) [Электронныйресурс] Режимдоступа / Online at stacks.iop.org/SST/26/105024.

15. Критская Т.В. Упругие напряжения в кремнии с внутренними геттерами / Т.В. Критская, В.Е. Кустов, Н.А. Трипачко, В.И. Шаховцов // Электронная техника. Сер. Материалы. -1989. -вып. 4. (241). - С.41-43. 16. Кустов В.Е. Влияние германия на внутренние упругие напряжения в кислородосодержащем кремнии / В.Е. Кустов, Т.В. Критская, Н.А. Трипачко, В.И. Шаховцов // ФТП. - 1988. -т.22.- вып. 2. - С.313-315. 
17. Кустов В.Е. Внутренние упругие деформации в кремнии / В.Е. Кустов, Т.В. Критская, Н.А. Трипачко, Л.И. Хируненко, В.И. Шаховцов, В.И. Яшник // Неорганические материалы. - 1991. - т.27, №6. - С. 1116-1118.

18. Критская Т.В., Быткин С.В., Радиационная деградация времени жизни неосновных носителей заряда в кремниевых p+-n-структурах. // Металургія. Збірник наукових праць Запорізької державної інженерної академії, вип. 1(26). - Запоріжжя, ЗДІА, 2012. - с.110-116

19. Вологдин Э.Н. Радиационные эффекты в интегральных микросхемах и методы испытаний изделий полупроводниковой электроники на радиационную стойкость [Учебное пособие по дисциплине «Радиационная стойкость изделий электронной техники»] / Э.Н. Вологдин, А.П. Лысенко. - М.: Научнообразовательный центр Московского региона в области фундаментальных проблем радиационной физики твердого тела и радиационного материаловедения. Московский государственный институт электроники и математики. -2002. - 46 с.

\section{REFERENCES}

1. Munaf Rahimo. Power Semiconductors for Power Electronics Applications ABB Switzerland Ltd, Semiconductors. CAS-PSI Special course Power Converters, Baden Switzerland, 8th May 2014, slides 43, 46.

2. Bytkin S.V. Silicon Doped with Germanium (n-Si<Ge $>$ ) Usage For Manufacturing Of Radiation Hardened Devices And Integrated Circuits. Fourth European Conference on Radiation and Its Effects on Components and Systems Proceedings, 1997. Cannes, France, pp. 141-146.

3. Byitkin S.V., Kritskaya T.V., Radin E.G., Goncharov V.I., Kunitskiy Yu.I., Kobeleva S.P. Eksperimentalnoe issledovanie harakteristik tiristorov, izgotovlennyih na $\mathrm{Si}<\mathrm{Ge}\rangle$, pri deystvii gamma-oblucheniya. Izvestiya vyisshih uchebnyih zavedeniy. Materialyi elektronnoy tehniki. \#3, 2012, str. 45-48.

4. S. V. Bytkin, T. V. Kritskaya, S. P. Kobeleva. Statistical analysis of germanium influence on radiation and thermal stability of the n-p-n-p device structures based on CZ-Si 〈P, Ge〉 electrophysical properties. Russian Microelectronics, December 2014, Volume 43, Issue 8, pp. 546-551.

5. S. Taranovich. Si vs. GaN vs. SiC: Which process and supplier are best for my power design? Сайт EDN, March 15, 2013. [Электронный ресурс] Режим доступа / http://www.edn.com/design/powermanagement/4409627/3/Si-vs--GaN-vs--SiC--Which-process-and-supplier-are-best-for-my-power-design.

6. Kritskaya T. V. Sovremennyie tendentsii polucheniya kremniya dlya ustroystv elektroniki: monografiya / T. V. Kritskaya; Zaporozh. gos. inzhener. akad. - Zaporozhe: ZGIA, 2013. - 353 c.

7. Crystal Growth Technology. [Электронный ресурс] Режим доступа / https://www.google.com.ua/url?sa=t\&rct=j\&q=\&esrc=s\&frm=1 \&source=web\&cd=2 \&cad=rja \&uact=8\&ved=0CC YQFjABahUKEwicjvyjgJrJAhXGCCwKHX4eBQQ\&url=https\%3A\%2F\%2Fwww.jsap.or.jp\%2Fenglish\%2Fimages\%2 Facademic_roadmap\%2Farm_e_09.pdf\&usg=AFQjCNGIxTwXP53r7IiRQ5U3f-

E_rvbK4A\&bvm=bv.107763241,d.bGg.

8. R.Pell. Power Week: Si-Based Power Discretes to Continue to Dominate Over Next Decade. Сайт EETimes 11/26/2014. [Электронный ресурс] Режим доступа / http://www.eetimes.com/document.asp?doc_id=1324777\&.

9. Kognitivnyiy vyizov i informatsionnyie tehnologii / G.G. Malinetskiy [i dr.] // Preprintyi IPM im. M.V. Keldyisha. 2010. \# 46. 28 s. URL: http://library.keldysh.ru/preprint.asp?id=2010-46.

10. Zhukova E.A. Hi-Tech: fenomen, funktsii, formyi. / Pod red. I.V. Melik -Gaykazyan. - Tomsk: Izdatelstvo Tomskogo gosudarstvennogo pedagogicheskogo universiteta, 2007. - $376 \mathrm{s.}$

11. G. Fisher, M. R. Seacrist, R. W. Standley. Silicon Crystal Growth and Wafer Technologies. ProceedingsoftheIEEE, Vol. 100, May 13th, 2012, 1454-1474.

12. Byitkin S. V., Kritskaya T.V. Modelirovanie protsessa nakopleniya radiatsionnyih defektov v protsesse oblucheniya monokristallov $\mathrm{Si}<\mathrm{P}>$ i Si $<\mathrm{P}, \mathrm{Ge}>$. MetalurgIya. ZbIrnik naukovih prats ZaporIzkoYi derzhavnoYi InzhenernoYi akademIYi, vip.1(21). - ZaporIzhzhya, 2010. - S.116-125.

13. BytkinS.V. Reduction of the Thermostable Radiation Defects Probability Formation in Si and SiGe as a Physical Basis of the Bipolar npn Transistors Radiation Hardness Increase at the Application of the Radiation \& Thermal Processing (RTP- technology). MAPLD 2004 Proceedings, September 8-10, 2004. The Johns Hopkins University- Applied Physics Laboratory-Laurel, Maryland, USA. [Электронный ресурс] Режим доступа / http://klabs.org/mapld04/papers/p/p138_bytkin_p.doc.

14. C.A. Londos, E. N. Sgourou, A. Chroneos, V. V. Emtsev. Carbon, oxygen and intrinsic defect interactions in germanium-doped silicon. Semicond. Sci. Technol. 26(2011) 105024 (7pр) [Электронныйресурс] Режимдоступа / Online at stacks.iop.org/SST/26/105024.

15. Kritskaya T.V. Uprugie napryazheniya v kremnii s vnutrennimi getterami / T.V. Kritskaya, V.E. Kustov, N.A. Tripachko, V.I. Shahovtsov // Elektronnaya tehnika. Ser. Materialyi. -1989. -vyip. 4. (241). - S.41-43.

16. Kustov V.E. Vliyanie germaniya na vnutrennie uprugie napryazheniya v kislorodosoderzhaschem kremnii / V.E. Kustov, T.V. Kritskaya, N.A. Tripachko, V.I. Shahovtsov // FTP. - 1988. -t.22.- vyip. 2. - S.313-315.

17. Kustov V.E. Vnutrennie uprugie deformatsii v kremnii / V.E. Kustov, T.V. Kritskaya, N.A. Tripachko, L.I. Hirunenko, V.I. Shahovtsov, V.I. Yashnik // Neorganicheskie materialyi. - 1991. - t.27, \#6. - S. 1116-1118. 
18. Kritskaya T.V., Byitkin S.V., Radiatsionnaya degradatsiya vremeni zhizni neosnovnyih nositeley zaryada v kremnievyih p -n-strukturah. // MetalurgIya. ZbIrnik naukovih prats ZaporIzkoYi derzhavnoYi InzhenernoYi akademIYi, vip. 1(26). - ZaporIzhzhya, ZDIA, 2012. - s.110-116

19. Vologdin E.N. Radiatsionnyie effektyi $\mathrm{v}$ integralnyih mikroshemah i metodyi ispyitaniy izdeliy poluprovodnikovoy elektroniki na radiatsionnuyu stoykost [Uchebnoe posobie po distsipline «Radiatsionnaya stoykost izdeliy elektronnoy tehniki»] / E.N. Vologdin, A.P. Lyisenko. - M.: Nauchno-obrazovatelnyiy tsentr Moskovskogo regiona $\mathrm{v}$ oblasti fundamentalnyih problem radiatsionnoy fiziki tverdogo tela i radiatsionnogo materialovedeniya. Moskovskiy gosudarstvennyiy institut elektroniki i matematiki. -2002. - $46 \mathrm{~s}$.

Received 29.01.18

\section{MODELING OF S-SHAPED ACCUMULATION PROCESS A- AND E-CENTERS IN ISOVALENT DOPED GERMANIUM SILICON IN STATISTICA AND MATHCAD ENVIRONMENT}

Silicon doped with an isovalent germanium admixture $(\mathrm{Si}<\mathrm{Ge}>$ or $\mathrm{SiGe}$ in English-language scientific and technical literature) has a number of physico-technological advantages in terms of ensuring the radiation resistance of npn and npnp structures. The use of such silicon is very promising for power electronics, since it is able to supplant currently used silicon of the traditional quality, doped with phosphorus $(\mathrm{Si}\langle\mathrm{P}\rangle$ ) due to its higher thermal stability and radiation resistance, which positively affects the quality of the devices manufactured on its basis. The aim of the work is to simulate nonlinear accumulation of basic radiation defects in silicon in the STATISTICA and MathCAD environment and to evaluate the possibility of using Ge as an impurity that significantly slows down the radiation degradation of Si. Nonlinear accumulation of the main radiation defects (Aand E- centers) in silicon, subjected to irradiation by $\alpha$-particles, investigated. This process was simulated in the STATISTICA and MathCAD environment. Shown, that doping of silicon with germanium makes it possible substantially slow down the radiation-induced degradation of silicon up to certain doses and use of such material for manufacturing of radiation-resistant semiconductor devices, in particular, multilayered structures of high-voltage power devices of the general industrial and transport electric drive.

Keywords: silicon alloying with germanium, nonlinear accumulation of basic radiation defects, manufacturing of radiation-resistant semiconductor devices.

\section{МОДЕЛЮВАННЯ S-ОБРАЗНОГО ПРОЦЕСУ НАКОПИЧЕННЯ А- I Е-ЦЕНТРІВ В ІЗОВАЛЕНТНОЮ ЛЕГОВАНИХ ГЕРМАНІЄМ КРЕМНІЇ В СЕРЕДОВИЩI STATISTICA I MATHCAD}

Досліджено нелінійне накопичення основних радіаційних дефектів (А- і Е- центрів) в кремнії, що піддається опроміненню $\alpha$-частинками. Проведено моделювання цього процесу в середовищі STATISTICA i MathCAD. Показано, що легування кремнію германієм дозволяє істотно сповільнити радіаційну деградацію кремнію в певному діапазоні доз опромінення і використовувати такий матеріал для виготовлення радіаційно-стійких напівпровідникових приладів, зокрема, багатошарових структур високовольтних силових приладів загальнопромислового і транспортного електроприводу.

Ключові слова: легування кремнію германієм, нелінійне накопичення основних радіаційних дефектів, виготовлення радіаційно-стійких напівпровідникових приладів

Быткин Сергей Витальевич - к.т.н., начальник отдела конъюнктурно-технологического системного анализа, ПАТ «Запорожсталь».

Bytkin Sergey - Candidate of Technical Sciences, Head of the conjuncture-technological systems analysis, Zaporozhstal JSC.

Критская Татьяна Владимировна - д.т.н., профессор, Запорожская государственная инженерная академия.

Kritskaya Tatyana - Doctor of Technical Sciences, Professor, Zaporizhzhya State Academy of Engineering. 